\title{
Pulmonary fibrosis: rate of disease progression as a trigger for referral for lung transplantation
}

\author{
Laura S Mackay, Rachel L Anderson, Gareth Parry, James Lordan, Paul A Corris, Andrew J Fisher
}

Thorax 2007;62:1069-1073. doi: 10.1136/thx.2006.068494

See end of article for authors' affiliations

Correspondence to: Dr Andrew J Fisher, Freeman Hospital, Newcastle upon Tyne NE7 7DN, UK; A.J.Fisher@ newcastle.ac.uk

Received 12 July 2006 Accepted 24 May 2007 Published Online First 15 June 2007

\begin{abstract}
Background: Lung transplantation is the only treatment modality that provides a survival advantage in pulmonary fibrosis, but many patients deemed suitable will die awaiting lung transplantation. While donor organ shortage undoubtedly contributes to this, late referral to the transplant centre may also play a role. This study investigates factors influencing the chance of patients with pulmonary fibrosis reaching lung transplantation.

Methods: A single-centre retrospective review of patient demographic data, assessment investigations and subsequent clinical outcomes was performed for patients with pulmonary fibrosis assessed for lung transplantation over a 5-year period.

Results: Between March 1999 and March 2004, 129 patients with pulmonary fibrosis underwent formal transplant assessment. Sixty-nine were accepted and listed for lung transplantation. Of these, 17 were transplanted, 37 died while waiting, 4 were removed from the list and 11 were still waiting at the conclusion of the study. The median waiting time on the list for those transplanted was 103 days (range 6-904) compared with 125 days (range 2-547) for those who died while on the list $(p=0.65)$. There was no significant difference in age, spirometry, total lung capacity, gas transfer measures or 6 min walk distance between those who died waiting and those transplanted. However, time from onset of symptoms to transplant assessment was significantly shorter in those who died on the waiting list (median 29 months (range 2-120)) than in those transplanted (median 46 months (range 6-204), $p=0.037$ ).

Conclusion: Patients with pulmonary fibrosis who died awaiting transplantation had similar disease severity at assessment as those who achieved transplantation. However, the interval between symptom onset and transplant referral was significantly shorter in those who died while on the waiting list, suggesting they had more rapidly progressive disease. The rate of disease progression appears to be a more sensitive indicator for transplantation referral than any single physiological measure of disease severity and should act as an important trigger for early transplant referral.
\end{abstract}

$P$ ulmonary fibrosis is a progressive debilitating restrictive lung disease which, in most cases, is fatal within 3-5 years of diagnosis. ${ }^{1-4}$ It is currently recommended that patients diagnosed with pulmonary fibrosis initially receive treatment with corticosteroids and immunosuppressive therapy. ${ }^{5}$ There is, however, no evidence that this improves survival or reduces the rate of disease progression. Lung transplantation is the only treatment modality that provides an actuarial survival advantage in this population. ${ }^{6}$ The progressive nature of this disease and the short interval between diagnosis and death make this therapeutic option available only to a limited number of younger patients. The transplant process begins with referral to a specialist transplant centre. Those without an absolute contraindication are thereafter admitted for detailed inpatient assessment. Patients accepted onto the active waiting list will wait on average 12-18 months for a suitable donor organ in the UK. ${ }^{7}$ Consequently, many patients never achieve transplantation and die while on the waiting list. While donor organ availability undoubtedly contributes to this, patients with pulmonary fibrosis have the highest waiting list mortality of all patients awaiting lung transplantation, ${ }^{8}$ and the unpredictable natural history of this disease-together with late referral for transplant assessment-may play an important role in this statistic.

Timely assessment is important in all patients under consideration for lung transplantation, none more so than those with pulmonary fibrosis in whom the window of opportunity for transplant may be as little as 22 months. ${ }^{9}$ Referral criteria suggest that all patients under the age of
65 years who are symptomatic and have failed to respond to steroid and immunosuppressive therapy should be considered for transplantation. ${ }^{10}$ More specifically, a vital capacity of $<60 \%$ predicted or transfer factor of $<50 \%$ predicted should trigger referral. Waiting list mortality has remained high despite these guidelines, and it has been suggested that they do not adequately allow for the unpredictable and progressive nature of this disease. New guidelines published recently have attempted to address this issue, and suggest that all patients with usual interstitial pneumonia (UIP), irrespective of vital capacity, or fibrotic non-specific interstitial pneumonia (NSIP) should be assessed. ${ }^{11}$ However, such criteria are broad and require firm histological diagnosis that may not be easily accessible to all referring clinicians. In view of this, we reviewed the experience at our centre over a 5-year period, comparing those who died while on the waiting list with those who reached transplantation. Our principal aim was to identify objective differences that may help refine referral criteria and subsequently impact on survival in this population.

\section{METHODS}

A retrospective review of all patients with pulmonary fibrosis who underwent formal inpatient assessment for lung transplantation at our centre between March 1999 and March 2004

Abbreviations: IIP, idiopathic interstitial pneumonia; IPF, idiopathic pulmonary fibrosis; NSIP, non-specific interstitial pneumonia; $\mathrm{PaO}_{2}, \mathrm{PaCO}_{2}$, arterial oxygen and carbon dioxide tensions; PASP, pulmonary artery systolic pressure; UIP, usual interstitial pneumonia 
was performed. Patient case notes were used to extract baseline demographic data, histopathological diagnosis, ABO blood group, body mass index and the results of pulmonary function tests, arterial blood gas measurement and unencouraged 6 min walk test distance. Patients were categorised as having idiopathic pulmonary fibrosis (IPF)/UIP or non-IPF because of the tendency of IPF/UIP to run a more rapidly progressive course. The diagnosis of IPF was based on the characteristic findings on biopsy and high-resolution CT scanning in those who died and from the explant pathology in those transplanted. In some cases where it was unclear which subtype of idiopathic interstitial pneumonia (IIP) was present, patients were assigned "unclassified IIP".

The clinical course of those listed for transplantation was followed thereafter until September 2004. In addition, we examined the length of time between each stage in the transplant process: (1) symptom onset to assessment; (2) assessment to listing; and (3) listing to transplantation.

Our aims were to identify objective differences in demographic measures, physiological measures and clinical course between those transplanted and those who died on the waiting list in order to refine referral criteria.

\section{Analysis of data}

All demographic and clinical data (excluding static lung volumes and transfer factor) are expressed as mean (SD). All remaining data are expressed as median (range). Statistical analysis was performed using the Student unpaired $t$ test for parametric data, Mann-Whitney $U$ test for non-parametric data, and $\chi^{2}$ test and Fisher exact test. (Microsoft Excel and Arcus Quickstat). A p value of $<0.05$ was considered significant.

\section{RESULTS}

One hundred and twenty-nine patients (92 men) of mean (SD) age 53 (7) years with pulmonary fibrosis, referred from 54 centres, underwent formal inpatient assessment between March 1999 and March 2004. Median time from symptom onset to first transplant assessment was 36 months (range 2240). Of these 129 patients, 69 (53\%) were listed for transplantation. Of the remaining 60 patients who were not listed, only 3 were deemed too unwell at the time of assessment. Thirty of the 60 not listed had significant comorbidities which acted as a contraindication to transplantation, the commonest of which were ischaemic heart disease and obesity. Nineteen of the 60 were deemed too well for transplantation at the time of assessment. Importantly, we found no significant difference in spirometry or static lung volumes, exercise capacity or degree of hypoxia between those listed and those not. However, those who were not listed had a significantly higher transfer factor, indicating that this group had less advanced disease.

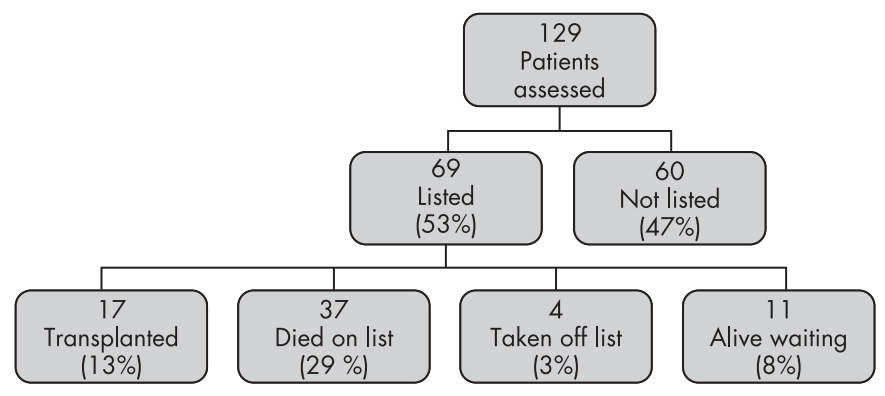

Figure 1 Flow chart showing outcomes for patients with pulmonary fibrosis undergoing formal lung transplant assessment during the 5-year period of the study.
Of the 69 patients listed for transplantation, 37 (54\%) died while on the waiting list while 17 (25\%) were transplanted. Eleven patients remained alive on the list at the end of the study and 4 were removed from the list (fig 1).

Fifteen $(88 \%)$ of those transplanted and 27 (73\%) of those who died while on the waiting list had a diagnosis of IPF/UIP based on pathology with or without radiology (table 1). In five patients who died we were unable accurately to assign a subtype of IIP and have classified them as "unspecified IIP". There was no significant difference in the proportions with IPF and non-IPF between those transplanted and those who died while on the waiting list $(\mathrm{p}=0.3$, Fisher exact test).

\section{Time course of assessment, listing and transplantation} The median time from symptom onset to initial lung transplantation assessment was significantly shorter in patients who died while on the waiting list than in those who underwent transplantation (29 months (range 2-120) vs 46 months (range 6-204); $p=0.037$, Mann-Whitney U test). There was no significant difference between patients who died on the waiting list and those who were transplanted in time from assessment to listing (median 60 days (range 4-998) vs 48 days (range $7-305) ; p=0.32$, Mann-Whitney $U$ test). The two groups spent similar lengths of time on the transplant waiting list (median 103 days (range 6-904) for those transplanted vs 125 days (range 2-547) for those who died on the waiting list; $\mathrm{p}=0.65$, Mann-Whitney $U$ test).

\section{Baseline demographic data}

There was no significant difference in age, 6 min walk distance, desaturation on walk test and body mass index between those who died while on the waiting list and those who underwent transplantation (table 2). An arterial blood gas analysis was performed on all patients at transplantation assessment. For clinical reasons it was necessary to perform this test in some while receiving supplementary oxygen. Therefore, in addition to comparing the degree of hypoxia between those transplanted and those who died while on the waiting list, we allocated patients receiving oxygen to have an arterial oxygen tension $\left(\mathrm{PaO}_{2}\right)<8 \mathrm{kPa}$ (indicating respiratory failure) and compared those with $\mathrm{PaO}_{2}<8 \mathrm{kPa}$ with those with $\mathrm{PaO}_{2}>8 \mathrm{kPa}$. There was no significant difference in $\mathrm{PaO}_{2}$ and $\mathrm{PaCO}_{2}$ (table 2) and in the proportion with $\mathrm{PaO}_{2}<8 \mathrm{kPa}$ and $>8 \mathrm{kPa}$ ( $\mathrm{p}=0.5, \chi^{2}$ test) between those transplanted and those who died while on the waiting list. Lung function was also compared as an objective marker of disease severity. There was no difference in mean percentage predicted forced expiratory volume in $1 \mathrm{~s}$ or vital capacity and no difference in median total transfer factor, transfer factor corrected for alveolar volume or total lung capacity between those transplanted and those who died while on the waiting list, among those who were able to complete reliable lung function testing (table 2 ).

In some cases patients were unable to complete full testing as illustrated in table 2 . We therefore investigated by $\chi^{2}$ analysis and Fisher exact tests whether there were significant differences in the proportion of patients transplanted and those who died while waiting in terms of who could and could not complete tests (table 3). No significant differences were found, except for the measurement of vital capacity where those transplanted were less likely to be able to perform this manoeuvre.

\section{Presence of pulmonary hypertension}

Echocardiograms were performed at assessment on all patients to investigate the incidence and degree of concomitant pulmonary hypertension. Adequate views in order to make an assessment of pulmonary artery pressure were available in 119 
Table 1 Diagnoses of transplanted patients and those who died while on the waiting list

\begin{tabular}{lll}
\hline Diagnosis & $\begin{array}{l}\text { Transplanted } \\
(\mathbf{n}=\mathbf{1 7})\end{array}$ & $\begin{array}{l}\text { Died on list } \\
(\mathbf{n}=\mathbf{3 7})\end{array}$ \\
\hline IPF/UIP & 15 & 27 \\
Based on & & \\
$\quad$ Biopsy/radiology & 15 & 13 \\
$\quad$ Radiology & 0 & 14 \\
Non-IPF/non-IIP & 2 & 10 \\
IIP unspecified & 0 & 5 \\
CT disease associated & 1 & 2 \\
Hypersensitivity pneumonitis & 1 & 3 \\
\hline
\end{tabular}

Patients are subdivided into those with idiopathic pulmonary fibrosis (IPF)/ usual interstitial pneumonia (UIP) and those with other forms of idiopathic interstitial pneumonia (IIP) and non-IIP forms of pulmonary fibrosis. Patients with IPF are classified according to findings on biopsy and radiology as illustrated above. Data for those transplanted were obtained from explant pathology.

cases (92\%). A pulmonary artery systolic pressure (PASP) of $>50 \mathrm{~mm} \mathrm{Hg}$ estimated via tricuspid regurgitant velocity was taken as evidence of pulmonary hypertension. Sixteen patients $(30 \%)$ had documented pulmonary hypertension. We found no significant difference in the presence or absence of pulmonary hypertension between those transplanted and those who died on the waiting list $\left(p=0.2, \chi^{2}\right.$ test). In addition, among those with documented pulmonary hypertension, there was no difference in severity (mean (SD) estimated PASP 59 (10) $\mathrm{mm} \mathrm{Hg}$ (7 patients transplanted) vs. 65 (11) $\mathrm{mm} \mathrm{Hg}$ (9 patients died on list) $(\mathrm{p}=0.3$, unpaired $t$ test $)$.

\section{Blood group}

Thirty-seven patients who were blood group $\mathrm{O}$ were listed, of whom only 5 were transplanted. In contrast, 9 of the 25 patients with blood group A received a transplant and 3 of 5 with blood group B were transplanted $\left(p=0.042, \chi^{2}\right.$ test). No patients with blood group $\mathrm{AB}$ received a transplant in this cohort.

\section{DISCUSSION}

Patients with pulmonary fibrosis have the highest waiting list mortality of all those awaiting lung transplantation, and new more sensitive triggers to determine the optimal timing of transplant referral are urgently needed. In this study we found that patients who died while on the transplant waiting list had similar disease severity based on spirometry, static lung volumes, gas transfer factor, degree of hypoxia, incidence of pulmonary hypertension and 6 min walk test to those who achieved transplantation. However, those who died waiting were referred earlier, with a significantly shorter interval between symptom onset and referral for transplantation assessment. These findings suggest that those who died on the waiting list reached the level requiring transplantation more rapidly. There was no significant difference between groups in time spent on the waiting list. From these observations, we believe that those who died on the waiting list had more rapidly progressive disease, with a shorter interval from symptoms to assessment, and subsequent continued rapid decline while on the waiting list. In further support of this theory, we found no significant difference in time between assessment and listing, and listing and transplantation (ie, days spent on the transplant list) between the two groups. Thus, delays in listing and longer wait times on the transplant list do not account for the excess mortality in those on the waiting list.

These findings are in agreement with a recently published smaller study that showed no difference in conventional physiological predictors but found a similar difference in time from diagnosis to referral. ${ }^{12}$ Timmer et al ${ }^{13}$ also noted a trend towards shorter time from diagnosis to referral for patients who died on the waiting list, but these differences (43.2 months among those alive on the waiting list vs 20.9 months among those who died) did not reach significance. These studies support our hypothesis that the rate of disease progression has an important role in determining the chance of achieving transplantation. Collard et al $^{14}$ showed that changes in dyspnoea score, total lung capacity, vital capacity and oxygen saturation over 6 months were predictive of survival and advocated that such change may provide more accurate prognostic information than baseline physiological measurements alone. Hanson and colleagues $^{15}$ reported that changes in vital capacity and single breath transfer factor after 1 year of treatment strongly predictive of survival time in patients with IPF. A 10\% reduction in vital capacity or $20 \%$ reduction in single breath transfer factor after 1 year predicted poorer survival than those patients with unchanged or improved tests. Data were, however, only compared at 1 year so the actual rate of physiological decline and the impact on survival remains unknown.

The pathological classification in pulmonary fibrosis is a most informative guide with regard to prognosis and an accurate diagnosis is always sought. ${ }^{16}$ Diagnosis has been refined by the new classification system of IIP. ${ }^{16}$ Patients were assigned a

Table 2 Baseline demographic and clinical data obtained at transplant assessment for those transplanted and those who died on the waiting list

\begin{tabular}{|c|c|c|c|c|c|}
\hline & $\begin{array}{l}\text { Transplanted } \\
(n=17)\end{array}$ & $\mathbf{n}$ & $\begin{array}{l}\text { Died on list } \\
(n=37)\end{array}$ & $\mathrm{n}$ & p Value \\
\hline Age (years) & $53(8)$ & 17 & $55(7)$ & 37 & 0.3 \\
\hline 6MWT distance $(\mathrm{m})$ & $226(106)$ & 16 & 199 (129) & 30 & 0.5 \\
\hline Desaturation on $6 \mathrm{MWT}(\%)$ & $79(10)$ & 15 & 74 (19) & 28 & 0.07 \\
\hline Body mass index & $25.5(5.3)$ & 17 & $26.7(3.9)$ & 37 & 0.4 \\
\hline $\mathrm{PaO}_{2}$ (kPa, room air) & $7.9(1.6)$ & 12 & $7.7(1.8)$ & 24 & 0.7 \\
\hline $\mathrm{PaCO}_{2}(\mathrm{kPa}$, room air) & $5.4(0.9)$ & 12 & $5.0(0.6)$ & 24 & 0.1 \\
\hline $\mathrm{FEV}_{1}(\%$ predicted $)$ & 47 (14) & 17 & $48(16)$ & 37 & 0.8 \\
\hline VC (\% predicted) & $43(17)$ & 12 & 44 (17) & 37 & 0.9 \\
\hline TLCO (\% predicted) ${ }^{*}$ & $25(16-36)$ & 13 & $21(10-104)$ & 21 & 0.2 \\
\hline Kco $(\% \text { predicted })^{*}$ & $60(18-96)$ & 13 & $50(22-91)$ & 21 & 0.2 \\
\hline TLC (\% predicted)* ${ }^{*}$ & $46(33-86)$ & 15 & $45(28-90)$ & 30 & 0.9 \\
\hline
\end{tabular}

Data shown are mean (SD) or *median (range).

$6 \mathrm{MWT}, 6$ min walk test; $\mathrm{PaO}_{2}, \mathrm{PaCO}_{2}$, arterial oxygen and carbon dioxide tensions; $\mathrm{FEV}_{1}$, forced expiratory volume in $1 \mathrm{~s}$; VC, vital capacity; TLCO, total lung transfer factor; KCO, transfer factor corrected for alveolar volume; TLC, total lung capacity.

Mean/median values were compared using the unpaired $t$ test or Mann-Whitney $U$ test to detect differences in severity of disease (significance, $\mathrm{p}<0.05$ ). 
Table 3 Comparison of number of patients in each group able to complete full baseline demographic and clinical data at assessment for those transplanted and those who died on the list using $\chi^{2}$ analysis and Fisher exact testing where appropriate $(p<0.05)$

\begin{tabular}{|c|c|c|c|}
\hline & $\begin{array}{l}\text { Patients transplanted who } \\
\text { completed the test }\end{array}$ & $\begin{array}{l}\text { Patients who died } \\
\text { awaiting transplantation } \\
\text { who completed the test }\end{array}$ & $\begin{array}{l}\text { p Value (those } \\
\text { completing tests } \\
\text { vs those not) }\end{array}$ \\
\hline 6MWT & 16 & 30 & 0.41 \\
\hline Desaturation on $6 \mathrm{MWT}$ & 15 & 28 & 0.47 \\
\hline $\mathrm{PaO}_{2}$ & 12 & 24 & 0.76 \\
\hline $\mathrm{PaCO}_{2}$ & 12 & 24 & 0.76 \\
\hline $\mathrm{FEV}_{1}$ & 17 & 37 & 1 \\
\hline VC & 12 & 37 & 0.002 \\
\hline TLCO & 13 & 21 & 0.23 \\
\hline KсO & 13 & 21 & 0.23 \\
\hline TLC & 15 & 30 & 0.70 \\
\hline
\end{tabular}

diagnosis of IPF based on clinical history and characteristic findings on high-resolution CT scanning and biopsy. However, as is frequently encountered in clinical practice, despite best efforts, some patients do not receive a firm diagnosis as evidenced by those with "unspecified IIP". Thus, even with greater correlation between histology and clinicoradiological findings, a firm diagnosis is not always reached even after biopsy. ${ }^{12}{ }^{17}$ The presence of another causative factor (such as connective tissue disease or exposure to asbestos) can make a correct diagnosis more elusive. In this study, two patients whose pretransplant diagnosis was connective tissue diseaseassociated fibrosis and two who were thought to have NSIP and desquamative interstitial pneumonia, respectively, were confirmed as having UIP when the pathology of their explanted lung was reviewed.

UIP is the commonest form of IIP and has the worst $\operatorname{prognosis}^{18}$; however, other forms are also referred for transplantation as witnessed in this study. Evidence suggests that the survival advantage conveyed by NSIP does not emerge until 18-24 months after diagnosis. ${ }^{19}$ Clinicians should not be falsely reassured by a diagnosis of NSIP as these patients can be misdiagnosed or present with rapidly progressive phenotypes that mimic UIP. Importantly, most patients referred for transplantation come from centres without specific expertise or services in fibrotic lung disease. The heterogeneous nature of this cohort of patients and, indeed, the underlying disease process therefore make our findings pertinent. The rate of physiological and symptomatic decline in any form of pulmonary fibrosis appears to provide a simple accessible tool that may be of greater prognostic value than the underlying pathological diagnosis in patients considered for transplantation.

$\mathrm{PaO}_{2}$ is reported to predict survival in this population, ${ }^{13}$ but we found no difference in the degree of hypoxia or the presence of respiratory failure (as defined by $\mathrm{PaO}_{2}<8 \mathrm{kPa}$ ) in our cohort, thus supporting our theory that patients who died while on the waiting list had similar disease severity to those transplanted. Similarly, the 6 min walk test has been shown to predict poorer survival, ${ }^{20}$ with a distance of $<207 \mathrm{~m}$ having a fourfold greater rate of mortality than those with a walk distance greater than this distance. In our study, while we found no significant difference between those transplanted and those who died while on the waiting list, those who died had a mean walk distance of $199 \mathrm{~m}$ which is in keeping with these findings. The performance of this test on supplemental oxygen may have allowed some to achieve higher than expected walk distances, thus masking this result.
The presence of pulmonary hypertension was shown to correlate with survival in patients with pulmonary fibrosis in a study by Nadrous and colleagues. ${ }^{21}$ They stratified patients into three groups depending on PASP ( $<35 \mathrm{~mm} \mathrm{Hg}, 36-50 \mathrm{~mm} \mathrm{Hg}$, $>50 \mathrm{~mm} \mathrm{Hg}$ ) and then followed them for 4.8, 4.1 and 0.8 years, respectively. Those with PASP $>50 \mathrm{~mm} \mathrm{Hg}$ had a significantly worse survival when analysed by the KaplanMeier method. In our study we found no difference in the presence or degree of pulmonary hypertension between those who died while on the waiting list and those transplanted. The measurements of PASP obtained by echocardiography may have been too insensitive to detect a difference between these two groups.

Importantly, $\mathrm{ABO}$ blood group did influence the chance of achieving transplantation with patients of blood groups $\mathrm{A}$ and $\mathrm{B}$ being more likely to receive a transplant. While this finding should not be directly used in referral criteria, it may have a role in the decision-making process at transplant assessment in group $\mathrm{O}$ patients who are moving beyond the transplant window of opportunity, directing treatment towards a palliative approach.

The use of explant lung pathology to establish classification of IIP in those transplanted is by definition limited to only one group. This may introduce bias. We took this strategy in order that those transplanted were not assumed to have forms of pulmonary fibrosis with a better prognosis than UIP/IPF.

Incomplete data are inevitable in this retrospective "real life" observational study of clinical practice. To investigate any confounding this might cause, we examined whether significant differences existed in the proportions of those able to complete tests and those not (ie, whether disease severity in either group was masked by incomplete data). We found no such difference, except for vital capacity, where those transplanted were less able to complete the manoeuvre. Thus, at assessment, we believe that all patients accepted onto the transplant waiting list had similar disease severity.

This retrospective review encounters the biases and limitations that feature in all such studies. The data were, however, collected prospectively during detailed inpatient assessment, with all tests performed over a 4-day period. We acknowledge that the data on time to referral depends on accurate referral information. Another limitation is the lack of serial spirometric and gas transfer measures, providing information on the actual rate of decline in lung function. These data are not currently available as patients on the transplant waiting list do not generally have serial measures of lung function performed routinely as such repeated tests were previously thought to be 
of limited value. It could also be considered that these observations simply reflect local practice in the UK, although recent changes in the organ allocation system in the USA make these observations internationally relevant.

In conclusion, we have shown that patients who died on the lung transplant waiting list were referred earlier than those who were transplanted. These two groups did not differ significantly in lung function indices, 6 min walk test or degree of hypoxia, suggesting that when assessed they had similar disease severity. We believe that those who died on the waiting list had more rapidly progressive disease as evidenced by the shorter time from symptom onset to assessment. This study suggests that the rate of progression of disease may provide an indication of when to refer patients with pulmonary fibrosis for lung transplantation assessment. Further work is now required to investigate the rate of change in these patients and to determine the magnitude and velocity required to trigger transplant referral.

\section{Authors' affiliations}

Laura S Mackay, Rachel L Anderson, Gareth Parry, James Lordan, Paul

A Corris, Andrew J Fisher, Cardiopulmonary Transplant Unit, Freeman

Hospital, Newcastle upon Tyne and Applied Immunobiology and

Transplantation Research Group, University of Newcastle, Newcastle upon Tyne, UK

Funding: LSM is a Clinical Research Associate funded by AstraZeneca. RLA is a Clinical Research Associate funded by an ERS fellowship. AJF is funded by a GlaxoSmithKline Senior Clinical Fellowship.

Competing interests: None

\section{REFERENCES}

1 Mapel DW, Hunt WC, Utton R, et al. Idiopathic pulmonary fibrosis: survival in population based and hospital based cohorts. Thorax 1998;53:469-76.

2 Carrington CB, Gaensler EA, Coutu RE. Natural history and treated course of usual and desquamative interstitial pneumonia. N Engl J Med 1978;298:801-9.

3 Turner-Warwick M, Burrows B, Johnson A. Cryptogenic fibrosing alveolitis: clinical features and their influence on survival. Thorax 1980;35:171-80.

4 Hubbard R, Johnston I, Britton J. Survival in patients with cryptogenic fibrosing alveolitis: a population based cohort study. Chest 1998;113:396-400.
5 Selman M, King TE, Pardo A. Idiopathic pulmonary fibrosis: prevailing and evolving hypotheses about its pathogenesis and implications for therapy. Ann Intern Med, 2001;134:136-51.

6 Thabut G, Mal H, Castier Y, et al. Survival benefit of lung transplantation for patients with idiopathic pulmonary fibrosis. J Thorac Cardiovasc Surg 2003;126:469-75

7 Anyanwu AC, Rogers CA, Murday AJ. Intrathoracic organ transplantation in the United Kingdom 1995-99: results from the UK cardiothoracic transplant audit. Heart 2002;87:449-54.

8 Hosenpud JD, Bennett LE, Keck BM, et al. Effect of diagnosis on survival benefit of lung transplantation for end-stage lung disease. Lancet 1998;351:24-7.

9 Mogulkoc N, Brutsche MH, Bishop PW, et al. Pulmonary function in idiopathic pulmonary fibrosis and referral for lung transplantation. Am J Respir Crit Care Med 2001; 164:103-8.

10 ASTP/ATS/ERS/ISHLT. Joint Statement. International guidelines for the selection of lung transplant candidates. Am J Respir Crit Care Med 1998;158:335-9.

11 Orens JB, Estenne M, Arcasoy S, et al. International guidelines for the selection of lung transplantation candidates: 2006 update. A consensus report from the Pulmonary Scientific Council of the International Society of Heart and Lung Transplantation. J Heart Lung Transplant 2006;25:745-55.

12 Reed A, Snell GI, Mclean C, et al. Outcomes of patients with interstitial lung disease referred for lung transplant assessment. Intern Med J 2006;36:423-30.

13 Timmer SJ, Karamzadeh AM, Yung GL, et al. Predicting survival of lung transplant candidates with idiopathic interstitial pneumonia. Does $\mathrm{PaO}_{2}$ predict survival? Chest 2002;122:779-84.

14 Collard HR, King TE, Bartelson BB, et al. Changes in clinical and physiologic variables predict survival in idiopathic pulmonary fibrosis. Am J Respir Crit Care Med 2003; 168:538-42

15 Hanson D, Winterbauer $\mathrm{RH}$, Kirtland $\mathrm{SH}$, et al. Changes in pulmonary function test results after 1 year of therapy as predictors of survival in patients with idiopathic pulmonary fibrosis. Chest 1995; 108:305-10.

16 Katzenstein AL, Myers JL. Idiopathic pulmonary fibrosis: clinical relevance of pathologic classification. Am J Respir Crit Care Med 1998;157:1301-15.

17 Flaherty KR, Travis WD, Colby TV, et al. Histopathologic variability in usual and non-specific interstitial pneumonias. Am J Respir Crit Care Med 2001;164:1722-7.

18 American Thoracic Society. Idiopathic pulmonary fibrosis: diagnosis and treatment. International consensus statement. American Thoracic Society (ATS) and the European Respiratory Society (ERS). Am J Respir Crit Care Med 2000;161:646-64.

19 Latsi PI, Du Bois RM, Nicholson AG, et al. Fibrotic idiopathic interstitial pneumonia: the prognostic value of longitudinal functional trends. Am J Respir Crit Care Med 2003;168:531-7.

20 Lederer DJ, Arcasoy SM, Wilt JS, et al. Six-minute walk distance predicts waiting list survival in idiopathic pulmonary fibrosis. Am J Respir Crit Care Med 2006;174:659-64.

21 Nadrous HF, Pellikka PA, Krowka MJ, et al. Pulmonary hypertension in patients with idiopathic pulmonary fibrosis. Chest 2005;128:2393-9. 